\title{
EARLY EFFECTS OF HIGH CHOLESTEROL DIET ON THE KIDNEY OF AN ANIMAL MODEL
}

Zenab B. Hamad Mohamed ${ }^{1}$, Hamad Abdulsalam Hamad Alfarisi ${ }^{1}$, Nor Zamzila Abdullah $^{1}$, Norra Harun ${ }^{2}$, Naznin Muhammad ${ }^{1}$ and Roslina Abdul Rahim ${ }^{1}$

${ }^{1}$ Kulliyyah of Medicine, International Islamic University Malaysia, ${ }^{2}$ Tengku Ampuan Afzan Hospital, Kuantan, Pahang, Malaysia.

Presenter: Zenab B. Hamad Mohamed, whiitefllower@gmail.com

Introduction: Previous studies have proven the existence of a complex association between progressive kidney damage and hypercholesterolemia. Most studies focused on the impact of chronic high blood cholesterol levels on the kidney. Information on the early effect of hypercholesterolemia on the kidney is still lacking. The aim of this study was therefore to determine early effect of high cholesterol diet on the kidney in an animal model.

Materials and method: Ten female Sprague-Dawley rats were divided into two groups. The control group was fed with commercial rat pellet while the high cholesterol diet (HCD) group was fed with $12 \%$ cholesterol diet and $0.3 \%$ cholic acid. Biochemical analysis (lipid profile and renal function) was performed at 48 hours, 7 days and 6 weeks of the experiment. The animals were sacrificed at 6 weeks and the kidneys were harvested for histological examination.

Results: The HCD group rats had significantly higher levels of serum total cholesterol (at 7 days and 6 weeks). The HDL-cholesterol and triglyceride levels were however lower at 6 weeks. The mean serum creatinine level of the HCD group were increased after 48 hours and 7 days compared to the control group. Histological examination of the kidney tissue of the HCD group at 6 weeks revealed segmental mesangial hypercellularity and mesangial matrix expansion of the glomeruli.

Conclusion: The $12 \%$ cholesterol diet induced dyslipidaemia in the animal model. It resulted in acute kidney injury based on the serum creatinine at 48 hours and 7 days. Kidney tissues examined at 6 weeks revealed changes confined to mesangial cells of the renal glomeruli. 